\title{
Survival of Japanese Patients with Pulmonary Arterial Hypertension after the Introduction of Endothelin Receptor Antagonists and/or Phosphodiesterase Type-5 Inhibitors
}

\author{
Seiichiro Sakao, Nobuhiro Tanabe, Yasunori Kasahara and Koichiro Tatsumi
}

\begin{abstract}
Objective Although endothelin receptor antagonists (ERAs) and phosphodiesterase type 5 (PDE5) inhibitors have become the most commonly used treatments for pulmonary arterial hypertension (PAH) since their introduction in 2005, it remains unknown whether these medications play a significant role in the survival of Japanese patients with PAH.

Methods The cardiac catheterization and survival data of 103 PAH patients were retrospectively reviewed. A comparison of survival benefits with regard to the type of PAH was completed in PAH patients diagnosed between 2005 and 2012 and those diagnosed between 1983 and 2004 and in patients undergoing treatment with ERAs and/or PDE5 inhibitors and those being treated with conventional therapy and/or oral beraprost. Although pulmonary vascular resistance (PVR) at baseline differed, the more recent group showed better survival rates compared with those observed in the early group (5-year survival: $70.1 \%$ vs. 44.8$)(p<0.05)$. In addition, the survival of PAH patients treated with ERAs and/or PDE5 inhibitors was superior to that of the patients treated without these medications (5- and 8-year survival: $77.8 \%$ and $66.7 \%$ vs. $39.0 \%$ and $37.0 \%$, respectively) $(\mathrm{p}<0.05)$, especially in patient with idiopathic and heritable PAH.

Conclusion Superior survival rates are observed in patients with idiopathic and heritable PAH after introduction of ERAs and PDE5 inhibitors, and the use of these drugs provides benefits for survival.
\end{abstract}

Key words: pulmonary arterial hypertension $(\mathrm{PAH})$, endothelin receptor antagonists (ERAs), phosphodiesterase type 5 (PDE5) inhibitors

(Intern Med 51: 2721-2726, 2012)

(DOI: 10.2169/internalmedicine.51.8162)

\section{Introduction}

An important pathological feature of pulmonary arterial hypertension $(\mathrm{PAH})$ is pulmonary vascular remodeling associated with marked proliferation of pulmonary artery endothelial cells (ECs) and/or smooth muscle cells (SMCs) as well as components of the extracellular matrix that results in the obstruction of blood flow in resistant pulmonary arteries $(1,2)$. Moreover, it appears that all of these conditions ultimately lead to signaling imbalances between vasoconstrictive (e.g., endothelin) and vasodilatory (e.g., prostacyclin and nitric oxide) compounds (3).

There are three classes of drugs approved for the evidence-based treatment of PAH (4): prostacyclin analogues (e.g., epoprostenol, beraprost, treprostinil and iloprost [treprostinil and iloprost are approved outside Japan]), endothelin receptor antagonists (ERAs) $(5,6)$ (e.g., ambrisentan and bosentan) and phosphodiesterase type 5 (PDE5) inhibitors (7) (e.g., sildenafil and tadalafil). These drugs, which are currently used for the treatment of PAH, act not only by opposing any abnormal vasoconstriction, but also by inhibiting the growth of normal SMC (2). Because the drugs currently approved to treat PAH are not curative, patients require long-term therapy. In addition, long-term use of these drugs may provide sustained benefits in terms of exercise capacity and pulmonary hemodynamics in comparison to placebos or historical controls in patients with PAH (3). 
Because of their availability and convenience, oral drugs (e.g., ambrisentan, bosentan, sildenafil and tadalafil) have recently become common treatments for PAH in Japan after being introduced in 2005. However, it remains to be elucidated whether these oral medications significantly improve survival in Japanese patients with PAH in comparison to conventional therapy.

Historically, the management of patients with PAH has been limited to conventional therapies such as anticoagulants, calcium channel blockers, diuretics, digoxin and supplemental oxygen. Beraprost was the first orally active and chemically stable prostacyclin analog to be developed and has been available for the treatment of PAH in Japan since 1992. Patients treated with beraprost demonstrate improvements in exercise capacity and symptoms within short-term durations (8). However, no beneficial effects of oral beraprost are observed on exercise capacity at nine or 12 months (9). This indicates that oral beraprost may not have sustained long-term effects. Therefore, this drug has a weak recommendation in the PAH evidence-based treatment algorithm (4) and has been approved only in Japan and Korea (10). In fact, because of its cost and availability, oral beraprost was the first-line therapy for PAH in Japan before the approval of ERAs and PDE5 inhibitors for the treatment of PAH. Therefore, in this study, the beneficial effects of ERAs and PDE5 inhibitors on survival were evaluated without regard to oral beraprost therapy.

The aim of this study was to investigate cumulative survival benefits with regard to types of $\mathrm{PAH}$ in patients with PAH after the introduction of ERAs and PDE5 inhibitors in comparison to those observed in patients treated with conventional therapy and/or oral beraprost.

\section{Materials and Methods}

\section{Study subjects}

From June 1983 to February 2012, 103 patients older than 15 years with PAH were treated at Chiba University Hospital. In all patients, the diagnosis of PAH was established using cardiac catheterization and based on a documented mean pulmonary arterial pressure $\geq 25 \mathrm{mmHg}$ and a pulmonary capillary wedge pressure or left ventricular enddiastolic pressure $\leq 15 \mathrm{mmHg}$. All patients were classified as Group 1.1 to 1.4 on the current Dana Point classification (11). The study exclusion criteria were: 1) distal chronic thromboembolic pulmonary hypertension; 2) pulmonary veno-occlusive disease (PVOD) and/or pulmonary capillary hemangiomatosis ( $\mathrm{PCH})$; 3) left heart disease; and 4) chronic pulmonary disease (11). Although six of the 103 patients were classified as being in WHO function class IV, only four patients were treated with intravenous infusion of epoprostenol. Three of these patients were treated with ERAs and/or PDE5 inhibitors before the introduction of epoprostenol. According to Japanese legislation, informed consent is not required for retrospective collection of data corresponding to current practice. However, the database was anonymized and complied with the restrictive requirements of the Ministry of Health, Labor and Welfare dedicated to privacy, information technology and civil rights in Japan. The Ethics Committees of Chiba University Hospital approved the study protocol.

\section{Efficacy measurements}

The subjects were retrospectively divided into two groups: those diagnosed between 1983 and $2004(n=66)$ and those diagnosed between 2005 and $2012(n=37)$, since the introduction of ERAs and PDE5 inhibitors in Japan occurred in 2005. The subjects were further separated into two groups: those treated with ERAs and/or PDE5 inhibitors $(n=36)$ and those treated with conventional therapy, including anticoagulants, calcium channel blockers, diuretics, digoxin, supplemental oxygen and/or oral beraprost and epoprostenol $(\mathrm{n}=$ 67). In this analysis, the most recent hemodynamic data obtained before treatment were investigated. For more detailed analyses, the subjects were divided into subgroups according to types of PAH. The survival status of all patients was followed on a yearly basis and at the end of the study. Five of the 103 patients were lost to follow-up. The date of initiation of ERAs and PDE5 inhibitors was selected as the starting point to determine the survival period for assessing the effects of the drugs. The survival rates were calculated for all patients and by subgroups using Kaplan-Meier estimates. The baseline demographic and hemodynamic data were investigated in all patients and by subgroups.

\section{Statistical analysis}

The data were analyzed using JMP 9.0.0 (Japanese version, SAS Institute Inc., Tokyo, Japan) and the Excel-Toukei 2010 software program (Social Survey Research Information Co., Ltd., Tokyo, Japan). All results are expressed as the mean \pm the SD for continuous variables and as the number or percentage for categorical variables. The baseline demographic and hemodynamic data were compared using unpaired Student's $t$-tests. The survival from all-cause death was estimated using the Kaplan-Meier method, and differences between groups were examined for significance using the log-rank test. Univariate and multivariate cox proportional hazards models were used to investigate the independent effects of the factors on survival. A p value of 0.05 was considered to be statistically significant.

\section{Results}

\section{Baseline characteristics}

One hundred and three patients with PAH were initially enrolled in this study (Table 1). The mean patient age was $46.9 \pm 15.0$ years (range: 15 to 75 ) with a $4: 1$ female to male ratio. Forty-four patients $(42.7 \%)$ were identified as having idiopathic and heritable PAH. The patients were followed for a mean period of $58.3 \pm 61.3$ months and a median of 
Table 1. Baseline Clinical Characteristics and Hemodynamics in the Patients with PAH

\begin{tabular}{|c|c|c|c|}
\hline & $1983-2012, n=103$ & $1983-2004, n=66$ & $2005-2012, n=37$ \\
\hline Female/male & $86 / 17$ & $53 / 13$ & $33 / 4$ \\
\hline Age(yrs) & $46.9 \pm 15.0$ & $46.0 \pm 15.4$ & $48.3 \pm 14.4$ \\
\hline Survivor, n (\%) & $50(47.6 \%)$ & $21(31.8 \%)$ & $29(78.4 \%)$ \\
\hline \multicolumn{4}{|l|}{ Type of PAH } \\
\hline Idiopathic and heritable PAH, $\mathrm{n}(\%)$ & $44(42.7 \%)$ & $31(47.0 \%)$ & $13(35.1 \%)$ \\
\hline PAH associated with connective tissue disease, $n(\%)$ & 39 (37.9\%) & $25(37.9 \%)$ & $14(37.8 \%)$ \\
\hline PAH associated with congenital heart disease, $n(\%)$ & $8(7.8 \%)$ & $3(4.5 \%)$ & $5(13.5 \%)$ \\
\hline PAH associated with portal hypertension, $\mathrm{n}(\%)$ & $12(11.7 \%)$ & $7(10.6 \%)$ & $5(13.5 \%)$ \\
\hline \multicolumn{4}{|l|}{ Hemodynamics } \\
\hline mRAP, mmHg & $5.2 \pm 6.1$ & $4.3 \pm 4.7$ & $6.9 \pm 7.8$ \\
\hline mPAP, $\mathrm{mmHg}$ & $47.9 \pm 13.2$ & $48.8 \pm 13.9$ & $46.2 \pm 11.7$ \\
\hline $\mathrm{mPCWP}, \mathrm{mmHg}$ & $6.4 \pm 3.0$ & $5.7 \pm 2.9$ & $7.6 \pm 2.7^{*}$ \\
\hline $\mathrm{CO}, \mathrm{L} / \mathrm{min}$ & $4.2 \pm 1.4$ & $3.9 \pm 1.2$ & $4.7 \pm 1.5^{*}$ \\
\hline $\mathrm{Cl}, \mathrm{L} / \mathrm{min}$ per $\mathrm{m}^{2}$ & $2.7 \pm 0.9$ & $2.5 \pm 0.8$ & $3.1 \pm 1.0^{*}$ \\
\hline PVR, dyne sec $\mathrm{cm}^{-5}$ & $898.7 \pm 504.6$ & $984.6 \pm 521.2$ & $754.8 \pm 446.1^{*}$ \\
\hline mSAP, mmHg & $89.3 \pm 14.9$ & $90.5 \pm 13.4$ & $87.2 \pm 17.2$ \\
\hline Heart rate, beats/min & $78.2 \pm 13.8$ & $79.3 \pm 13.9$ & $76.4 \pm 13.8$ \\
\hline $\mathrm{SvO}_{2}, \%$ & $68.4 \pm 8.6$ & $68.3 \pm 9.0$ & $68.5 \pm 8.0$ \\
\hline \multicolumn{4}{|l|}{ WHO functional class } \\
\hline $\mathrm{I}, \mathrm{n}(\%)$ & $1(1.2 \%)$ & $0(0 \%)$ & $1(2.7 \%)$ \\
\hline II, n (\%) & $32(37.2 \%)$ & $17(32.7 \%)$ & $15(40.5 \%)$ \\
\hline III, n (\%) & 47 (54.7\%) & $30(57.7 \%)$ & $17(46.0 \%)$ \\
\hline IV, n (\%) & $6(7.0 \%)$ & $5(9.6 \%)$ & $1(2.7 \%)$ \\
\hline \multicolumn{4}{|l|}{ Treatment } \\
\hline Bosentan, n (\%) & $22(21.4 \%)$ & $8(12.1 \%)$ & $14(37.8 \%)$ \\
\hline Ambrisentan, $n(\%)$ & $1(1.0 \%)$ & $0(0.0 \%)$ & $1(2.7 \%)$ \\
\hline Sildenafil, n (\%) & $20(19.4 \%)$ & $3(4.5 \%)$ & $17(45.9 \%)$ \\
\hline Tadalafil, n (\%) & $2(1.9 \%)$ & $0(0.0 \%)$ & $2(5.4 \%)$ \\
\hline Epoprostenol, n (\%) & $4(3.9 \%)$ & $3(4.5 \%)$ & $1(2.7 \%)$ \\
\hline Oral beraprost, $\mathrm{n}(\%)$ & $36(35.0 \%)$ & $20(30.3 \%)$ & $16(43.2 \%)$ \\
\hline
\end{tabular}

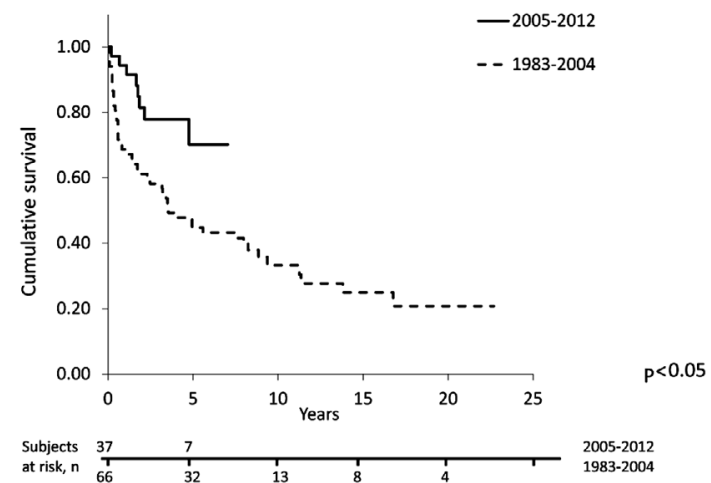

Figure 1. The Kaplan-Meier survival estimates for the PAH patients. The survival rate for patients treated between 2005 and 2012 (solid line) was $70.1 \%$ at 5 -years compared with 44.8\% for patients treated between 1983 and 2004 (dashed line; $\mathbf{p}<0.05$ by the Cox-Mantel log-rank test).

41.5 \pm 61.3 months (range: 1 to 276 ). The mean pulmonary arterial pressure (PAP) and mean pulmonary vascular resistance $(\mathrm{PVR})$ were $47.9 \pm 13.2 \mathrm{mmHg}$ and $898.7 \pm 504.6$ dyne. sec. $\mathrm{cm}^{-5}$, respectively.

\section{Survival}

Patients diagnosed between 1983 and $2004(n=66)$ vs. those diagnosed between 2005 and $2012(n=37)$

We divided the patients into two groups based on the timing of diagnosis (Table 1). We found that the patients diagnosed between 2005 and 2012 had a better survival rate than the patients diagnosed between 1983 and 2004 (5-year survival: $70.1 \%$ vs. $44.8 \%)(\mathrm{p}<0.05)$ (Fig. 1). However, cardiac output (CO) and pulmonary vascular resistance (PVR) at baseline significantly differed between the two groups (Table 1), and these differences make it difficult to attribute the superior outcome to the introduction of ERAs and PDE5 inhibitors.

\section{Outcomes of patients treated with ERAs and/or PDE5 inhibitors $(n=36)$ and those treated with con- ventional therapy and/or oral beraprost $(n=67)$}

To elucidate the absolute benefits of ERAs and/or PDE5 inhibitors on the survival of Japanese patients with PAH, an analysis was completed comparing the results of patients treated with ERAs and/or PDE5 inhibitors $(n=36)$ and the 
Table 2. Baseline Clinical Characteristics and Hemodynamics in the Patients with PAH

\begin{tabular}{|c|c|c|c|c|c|c|}
\hline & \multicolumn{2}{|l|}{$\begin{array}{l}\text { The PAH patients, } \\
n=106\end{array}$} & \multicolumn{2}{|c|}{$\begin{array}{l}\text { The idiopathic and heritable PAH } \\
\text { patients, } \\
\mathrm{n}=44\end{array}$} & \multicolumn{2}{|c|}{$\begin{array}{l}\text { The associated PAH patients, } \\
n=59\end{array}$} \\
\hline & $\begin{array}{l}\text { ERA and/or PDE5 } \\
\text { Inhibitors therapy, } \\
n=36\end{array}$ & $\begin{array}{l}\text { PAH therapies } \\
\text { without ERA and } \\
\text { PDE5 inhibitor, } \\
n=67\end{array}$ & $\begin{array}{l}\text { ERA and/or PDE5 } \\
\text { Inhibitors therapy, } \\
n=16\end{array}$ & $\begin{array}{l}\text { PAH therapies } \\
\text { without ERA and } \\
\text { PDE5 inhibitor, } \\
n=28\end{array}$ & $\begin{array}{l}\text { ERA and/or PDE5 } \\
\text { Inhibitors therapy, } \\
n=20\end{array}$ & $\begin{array}{l}\text { PAH therapies } \\
\text { without ERA and } \\
\text { PDE5 inhibitor, } \\
n=39\end{array}$ \\
\hline Female/male & $33 / 3$ & $53 / 14$ & $13 / 3$ & $19 / 9$ & $20 / 0$ & $34 / 5$ \\
\hline Age(yrs) & $47.3 \pm 14.4$ & $46.7 \pm 15.5$ & $44.8 \pm 14.0$ & $41.7 \pm 15.2$ & $49.2 \pm 14.7$ & $50.2 \pm 14.9$ \\
\hline Survivor, n (\%) & $28(69.4 \%)$ & $21(29.9 \%)$ & $14(87.5 \%)$ & $5(17.9 \%)$ & $14(70.0 \%)$ & $16(41.0 \%)$ \\
\hline \multicolumn{7}{|l|}{ Hemodynamics } \\
\hline $\mathrm{mRAP}, \mathrm{mmHg}$ & $5.5 \pm 7.9$ & $5.1 \pm 4.9$ & $3.9 \pm 2.7$ & $5.3 \pm 6.6$ & $6.8 \pm 410.2$ & $5.0 \pm 3.4$ \\
\hline $\mathrm{mPAP}, \mathrm{mmHg}$ & $44.9 \pm 11.8$ & $49.5 \pm 13.7$ & $44.4 \pm 11.1$ & $54.5 \pm 16.7^{*}$ & $45.3 \pm 12.6$ & $46.2 \pm 10.1$ \\
\hline $\mathrm{mPCWP}, \mathrm{mmHg}$ & $6.7 \pm 2.4$ & $6.2 \pm 3.2$ & $6.6 \pm 2.5$ & $6.0 \pm 3.4$ & $6.8 \pm 2.4$ & $6.4 \pm 3.2$ \\
\hline $\mathrm{CO}, \mathrm{L} / \mathrm{min}$ & $4.4 \pm 1.1$ & $4.1 \pm 1.5$ & $4.4 \pm 0.9$ & $3.7 \pm 1.4$ & $4.4 \pm 1.3$ & $4.4 \pm 1.6$ \\
\hline $\mathrm{Cl}, \mathrm{L} / \mathrm{min}$ per $\mathrm{m}^{2}$ & $2.9 \pm 0.7$ & $2.7 \pm 1.0$ & $2.7 \pm 0.5$ & $2.4 \pm 0.8$ & $3.0 \pm 0.9$ & $2.8 \pm 1.1$ \\
\hline PVR, dyne sec $\mathrm{cm}^{-5}$ & $771.4 \pm 440.3$ & $971.4 \pm 527.6^{*}$ & $736.7 \pm 338.9$ & $1166.7 \pm 609.7 *$ & $799.2 \pm 514.4$ & $842.9 \pm 427.2$ \\
\hline $\mathrm{mSAP}, \mathrm{mmHg}$ & $88.8 \pm 14.2$ & $89.6 \pm 15.3$ & $84.4 \pm 14.9$ & $89.2 \pm 14.1$ & $92.3 \pm 12.9$ & $89.9 \pm 16.3$ \\
\hline Heart rate, beats/min & $75.9 \pm 12.7$ & $79.6 \pm 14.3$ & $73.1 \pm 13.3$ & $83.0 \pm 14.3$ & $78.1 \pm 12.1$ & $77.2 \pm 14.0$ \\
\hline $\mathrm{SvO}_{2}, \%$ & $68.1 \pm 7.7$ & $68.5 \pm 9.1$ & $67.5 \pm 6.1$ & $66.1 \pm 9.5$ & $68.7 \pm 8.9$ & $70.1 \pm 8.7$ \\
\hline \multicolumn{7}{|l|}{ WHO functional class } \\
\hline I, n (\%) & $0(0.0 \%)$ & $1(1.5 \%)$ & $0(0.0 \%)$ & $0(0.0 \%)$ & $0(0.0 \%)$ & $1(2.6 \%)$ \\
\hline II, n (\%) & $15(41.7 \%)$ & $16(23.9 \%)$ & $6(37.5 \%)$ & $5(17.9 \%)$ & $9(45.0 \%)$ & $11(28.2 \%)$ \\
\hline III, n (\%) & $12(33.3 \%)$ & $32(47.8 \%)$ & $8(50.0 \%)$ & $14(50.0 \%)$ & $7(35.0 \%)$ & $18(46.2 \%)$ \\
\hline IV, n (\%) & $1(2.8 \%)$ & $5(7.5 \%)$ & $0(0.0 \%)$ & $3(10.7 \%)$ & $1(5.0 \%)$ & $2(5.1 \%)$ \\
\hline \multicolumn{7}{|l|}{ Treatment } \\
\hline Bosentan, n (\%) & $22(61.1 \%)$ & $0(0.0 \%)$ & $11(68.8 \%)$ & $0(0.0 \%)$ & $11(55.0 \%)$ & $0(0.0 \%)$ \\
\hline Ambrisentan, $\mathrm{n}(\%)$ & $1(2.8 \%)$ & $0(0.0 \%)$ & $1(6.3 \%)$ & $0(0.0 \%)$ & $0(0.0 \%)$ & $0(0.0 \%)$ \\
\hline Sildenafil, n (\%) & $18(50.0 \%)$ & $0(0.0 \%)$ & $7(43.8 \%)$ & $0(0.0 \%)$ & $11(55.0 \%)$ & $0(0.0 \%)$ \\
\hline Tadalafil, n (\%) & $2(5.6 \%)$ & $0(0.0 \%)$ & $1(6.3 \%)$ & $0(0.0 \%)$ & $1(5.0 \%)$ & $0(0.0 \%)$ \\
\hline Epoprostenol, n (\%) & $3(8.3 \%)$ & $1(1.5 \%)$ & $3(18.8 \%)$ & $0(0.0 \%)$ & $0(0.0 \%)$ & $1(2.6 \%)$ \\
\hline Oral beraprost, n (\%) & $14(38.9 \%)$ & $22(32.8 \%)$ & $7(43.8 \%)$ & $8(28.6 \%)$ & $7(35.0 \%)$ & $14(35.9 \%)$ \\
\hline
\end{tabular}

*p $<0.05$; vs ERA and/or PDE5 Inhibitors therapy

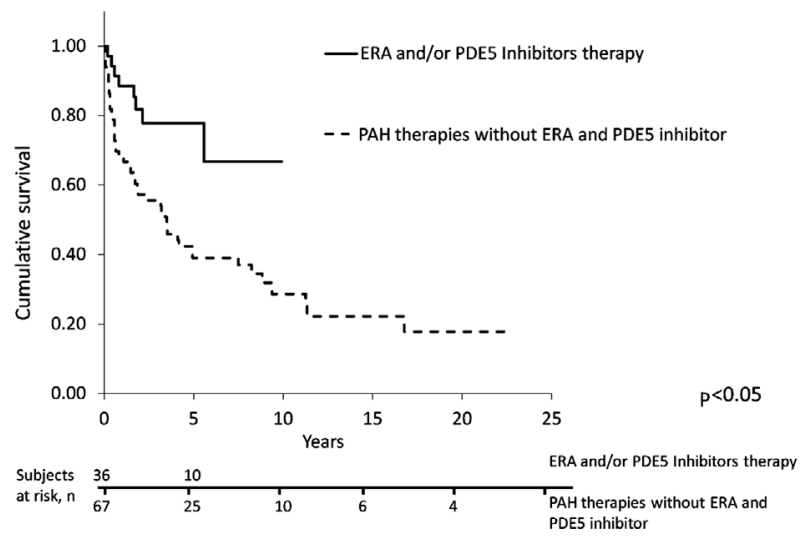

Figure 2. The Kaplan-Meier survival estimates for the PAH patients. The survival rates for patients treated with ERA and/or PDE5 inhibitor therapy (solid line) were $\mathbf{7 7 . 8 \%}$ and $66.7 \%$ at five and eight years compared with $39.0 \%$ and $37.0 \%$ for patients treated with PAH therapies without ERA or PDE5 inhibitors (dashed line; $\mathbf{p}<0.05$ by the Cox-Mantel log-rank test).

results of patients treated without ERAs and/or PDE5 inhibitors $(\mathrm{n}=67)$ (Table 2). A significant difference was observed between the two groups in the Kaplan-Meier survival curve (77.8\% and $66.7 \%$ vs. $39.0 \%$ and $37.0 \%$, respectively) $(\mathrm{p}<$ 0.05) (Fig. 2) and in PVR (Table 2). In particular, the patients with idiopathic and heritable PAH treated with ERAs and/or PDE5 inhibitors $(n=16)$ showed significantly better survival outcomes than those not treated with these drugs $(\mathrm{n}=28)$ (Table 2) (5- and 8-year survival: $92.9 \%$ and $69.6 \%$ vs. $26.0 \%$ and $20.8 \%$, respectively) (p<0.05) (Fig. 3). However, in the associated PAH patients (Table 2), no significant differences were observed between the groups (Fig. 4).

A univariate Cox proportional hazard analysis showed that cardiac index $(\mathrm{CI})$, mean pulmonary arterial pressure (mPAP) and the use of ERA and/or PDE5 inhibitor therapy were associated with cumulative survival. Moreover, CI and ERA and/or PDE5 therapy were the significant predictors of survival in the multivariate analysis. The use of ERA and/or PDE5 inhibitor therapy was an independent predictor for superior outcomes (Table 3).

\section{Discussion}

The data presented here show that the patients treated be- 
Table 3. Univariate and Multivariate Cox Proportional Hazards Models for the Predictors of Survival

\begin{tabular}{lllll}
\hline \multirow{2}{*}{ Factors } & \multicolumn{3}{l}{ Univariate } & \multicolumn{3}{c}{ Multivariate } \\
\cline { 2 - 5 } & $\mathrm{HR}(95 \% \mathrm{Cl})$ & $\mathrm{p}$ & $\mathrm{HR}(95 \% \mathrm{Cl})$ & $\mathrm{p}$ \\
\hline $\mathrm{Cl}\left(\mathrm{L} / \mathrm{min}\right.$ per m $\left.{ }^{2}\right)$ & $0.56(0.423-0.747)$ & 0.0001 & $0.60(0.458-0.810)$ & 0.0008 \\
$\mathrm{mPAP}(\mathrm{mmHg})$ & $1.03(1.005-1.048)$ & 0.0160 & $1.02(0.992-1.038)$ & 0.1875 \\
ERA and/or PDE5 Inhibitors therapy & $0.32(0.137-0.636)$ & 0.0008 & $0.39(0.168-0.796)$ & 0.0084 \\
(vs. PAH therapies without ERA and PDE5 inhibitor) & & & & \\
\hline
\end{tabular}

ERAs: endothelin receptor antagonists, PDE5: phosphodiesterase type 5 (PDE5)

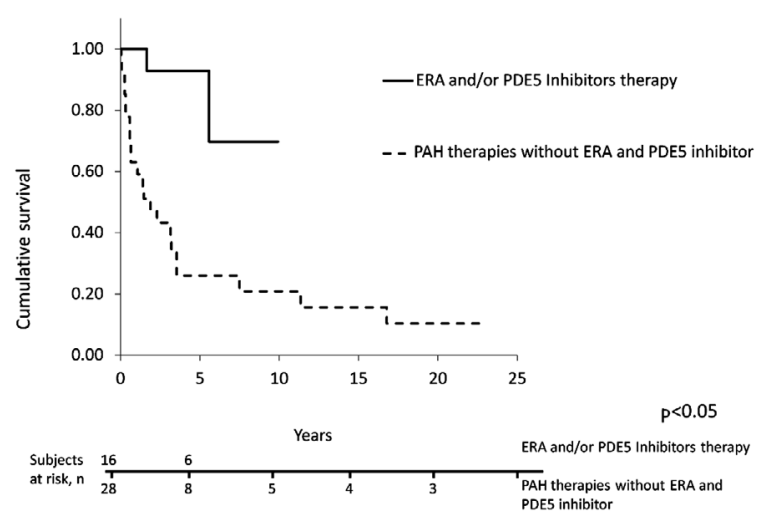

Figure 3. The Kaplan-Meier survival estimates for the idiopathic and heritable PAH patients. The survival rates for patients treated with ERA and/or PDE5 inhibitor therapy (solid line) were $92.9 \%$ and $69.6 \%$ at five and eight years compared with $\mathbf{2 6 . 0 \%}$ and $\mathbf{2 0 . 8 \%}$ for patients treated with PAH therapies without ERA or PDE5 inhibitors (dashed line; $\mathbf{p}<0.05$ by the Cox-Mantel log-rank test).

tween 2005 and 2012 had a better survival rate $(\mathrm{p}<0.05)$ (Fig. 1); however, these patients had significantly less serious hemodynamic alterations (Table 1). The hemodynamic difference observed between the groups makes it difficult to attribute the superior outcome to the introduction of ERAs and PDE5 inhibitors (Fig. 1).

Although it is beyond the scope of this paper to argue delaying the diagnosis, the presence of less serious hemodynamic alterations in the patients diagnosed between 2005 and 2012 suggests that earlier detection of PAH in patients with mild/moderate hemodynamic changes was achieved more often in that group than in the group diagnosed between 1983 and 2004. Since signs and symptoms of PAH do not generally manifest until hemodynamic changes are advanced, there are significant delays in diagnosing this disease. Although primary pulmonary hypertension (PPH) registry data from 1987 show that, at that time, the time from symptom onset to diagnosis with catheterization was 2.3 years (12), there have not been any recent advances in the diagnostic processes. The registry to evaluate early and long-term PAH (REVEAL) study showed that the average time from onset to diagnosis is still more than two years (13). The advances observed in this study may be

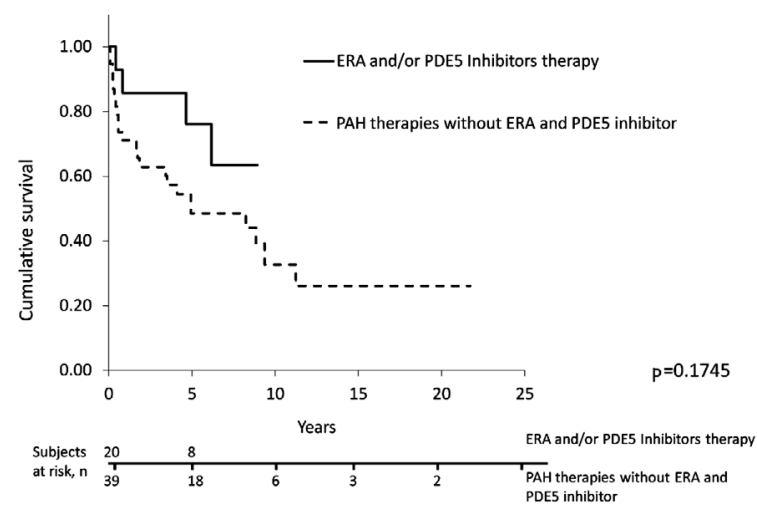

Figure 4. The Kaplan-Meier survival estimates for the associated PAH patients. The survival rates for patients treated with ERA and/or PDE5 inhibitor therapy (solid line) were 76.2\% and $63.5 \%$ at five and eight years compared with 48.5\% and $32.6 \%$ for patients treated with PAH therapies without ERA or PDE5 inhibitors (dashed line; $p=0.1745$ by the Cox-Mantel log-rank test).

based on the development and approval of oral therapies and increased doctor recognition of PAH following the introduction of these drugs.

This single-center, uncontrolled study demonstrated that idiopathic and heritable PAH patients treated with ERAs and/or PDE5 inhibitors $(n=13)$ have a higher survival rate than those treated with conventional therapy and/or oral beraprost (Fig. 3, Table 2). In line with previous reports (3), this result may support the concept that the use of these drugs provides benefits for the survival of Japanese patients with PAH. Nevertheless, the benefits of ERAs and/or PDE5 inhibitors on survival may be restricted to idiopathic and heritable PAH patients because no significant differences were observed between the groups in the associated PAH patients (Table 2, Fig. 4).

Because oral beraprost has a weak recommendation in the PAH evidence-based treatment algorithm (4) and has so far only been approved in Japan and Korea (10), this study was conducted without regard to oral beraprost therapy. However, this is a limitation of this study. It is impossible to deny that oral beraprost has a beneficial effect on the treatment of PAH. A randomized and properly controlled doseresponse study of beraprost is currently underway (14). 
There may be beneficial effects of high-dose oral beraprost on exercise capacity and hemodynamics in patients with PAH.

Epoprotenol was approved in Japan in 1999, and 23 patients treated after 1999 died in our center. In this study, only four patients (three between 1983 and 2004 and one between 2005 and 2012) were treated with epoprostenol and all survived. Although epoprotenol is strongly recommended by the WHO/NYHA class IV according to recent guidelines, we were unable to administer intravenous treatments in some of the 23 non-survivor cases because the patients were elderly ( $>70$ years of age) $(n=2)$, had comorbidities $(n=5)$ or were unwilling $(n=6)$ to undergo intravenous treatments. However, the early administration of epoprostenol therapy is suggested to improve survival in patients with a PVR $>1,000$ dynes.sec.cm ${ }^{-5}$.

The data presented in this study were limited because this was an observational study from a single center and the PAH patients were not treated in a randomized manner according to hemodynamics and comorbidities, i.e., this study included patients treated with conventional therapy, which may have favorably biased the results. We realize the limitations of interpreting our results. We interpreted the results of this study as part of a hypothesis-generating analysis, which suggested that there are beneficial effects of treatment with ERAs and/or PDE5 inhibitors on overall survival in idiopathic and heritable PAH patients. This hypothesis will need to be further investigated in a large confirmatory longterm trial in the future.

This study has evolved over the 28-year time period of our practice. The results of six minute walk distance (6 MWD) tests and brain natriuretic peptide (BNP) tests were not obtained as consistently in the past as they have been in the most recent eight years. For this reason, in this observational study, we evaluated survival benefits only in PAH patients treated with PAH-specific therapy in comparison to patients treated with conventional therapy, instead of comparing the 6MWD and BNP results.

In conclusion, this study suggests that superior survival rates are observed in patients with idiopathic and heritable PAH after the introduction of ERAs and PDE5 inhibitors, and the use of these drugs provides a survival benefit in patients with idiopathic and heritable PAH.

\section{Author's disclosure of potential Conflicts of Interest (COI).}

Tatsumi K: Honoraria, Glaxo Smith Kline and Actelion Pharmaceutical Ltd. Tanabe N: Honoraria, Actelion, Glaxo Smith Kline, Astellas and Pfizer.
Grant support: This study was supported by Research Grants from the Respiratory Failure Research Group, the Cardiovascular Diseases (19-9) and Research on Intractable Diseases (22-33) from the Ministry of Health, Labor and Welfare, Japan, a Grantin-Aid for Scientific Research (Category C 22590851) from the Japanese Ministry of Education, Culture, Sports Science and Technology, and the Takeda Science Foundation.

\section{References}

1. Humbert M, Morrell NW, Archer SL, et al. Cellular and molecular pathobiology of pulmonary arterial hypertension. J Am Coll Cardiol 43: 13S-24S, 2004.

2. Humbert M, Sitbon O, Simonneau G. Treatment of pulmonary arterial hypertension. N Engl J Med 351: 1425-1436, 2004.

3. Gomberg-Maitland M, Dufton C, Oudiz RJ, Benza RL. Compelling evidence of long-term outcomes in pulmonary arterial hypertension? A clinical perspective. J Am Coll Cardiol 57: 1053-1061, 2011.

4. Barst RJ, Gibbs JS, Ghofrani HA, et al. Updated evidence-based treatment algorithm in pulmonary arterial hypertension. J Am Coll Cardiol 54: S78-S84, 2009.

5. Yanagisawa M, Kurihara H, Kimura S, et al. A novel potent vasoconstrictor peptide produced by vascular endothelial cells. Nature 332: 411-415, 1988.

6. Channick RN, Sitbon O, Barst RJ, Manes A, Rubin LJ. Endothelin receptor antagonists in pulmonary arterial hypertension. $\mathrm{J}$ Am Coll Cardiol 43: 62S-67S, 2004.

7. Galiè N, Ghofrani HA, Torbicki A, et al. Sildenafil Use in Pulmonary Arterial Hypertension (SUPER) Study Group. Sildenafil citrate therapy for pulmonary arterial hypertension. $\mathrm{N}$ Engl $\mathrm{J}$ Med 353: 2148-2157, 2005.

8. Galie N, Humbert M, Vachiery JL, et al. Arterial Pulmonary Hypertension and Beraprost European (ALPHABET) Study Group. Effects of beraprost sodium, an oral prostacyclin analogue, in patients with pulmonary arterial hypertension: a randomized, doubleblind, placebo-controlled trial. J Am Coll Cardiol 39: 1496-1502, 2002.

9. Barst RJ, McGoon M, McLaughlin V, et al; Beraprost Study Group. Beraprost therapy for pulmonary arterial hypertension. $\mathbf{J}$ Am Coll Cardiol 41: 2119-2125, 2003.

10. Mubarak KK. A review of prostaglandin analogs in the management of patients with pulmonary arterial hypertension. Respir Med 104: 9-21, 2010.

11. Simonneau G, Robbins IM, Beghetti M, et al. Updated clinical classification of pulmonary hypertension. J Am Coll Cardiol 54: S 43-S54, 2009.

12. Rich S, Dantzker DR, Ayres SM, et al. Primary pulmonary hypertension: a national prospective study. Ann Intern Med 107: 216223, 1987.

13. REVEAL registry, Available at http://www.revealregistry.com. Accessed February 24, 2009.

14. Information source: Lung Rx Information obtained from ClinicalTrials.gov. Available at http://clinicaltrials.gov/ct2/home

(C) 2012 The Japanese Society of Internal Medicine http://www.naika.or.jp/imonline/index.html 